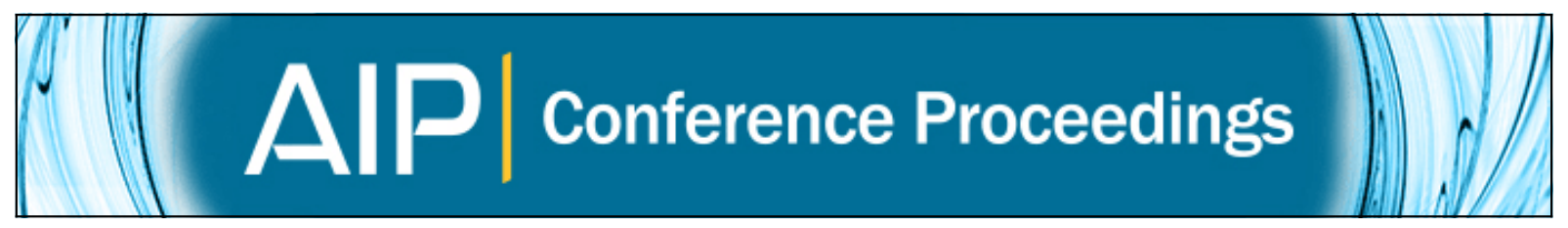

Development of an equipment for real time MTF measurement of optical systems

Dimas Rodrigues Romano, Sergio Antonio de Almeida Nobre, and Bráulio Fonseca Carneiro de Albuquerque

Citation: AIP Conference Proceedings 992, 882 (2008); doi: 10.1063/1.2926989

View online: http://dx.doi.org/10.1063/1.2926989

View Table of Contents: http://scitation.aip.org/content/aip/proceeding/aipcp/992?ver=pdfcov

Published by the AIP Publishing

Articles you may be interested in

Theoretical and experimental study on two-stage-imaging microscopy using ellipsometric contrast for real-time visualization of molecularly thin films

Rev. Sci. Instrum. 84, 053704 (2013); 10.1063/1.4804633

Validity of the line-pair bar-pattern method in the measurement of the modulation transfer function (MTF) in megavoltage imaging

Med. Phys. 35, 270 (2008); 10.1118/1.2816108

Slit design for efficient and accurate MTF measurement at megavoltage $\mathrm{x}$-ray energies

Med. Phys. 34, 1535 (2007); 10.1118/1.2717405

Modeling and measurement of the detector presampling MTF of a variable resolution x-ray CT scanner Med. Phys. 34, 1062 (2007); 10.1118/1.2436977

Validation of MTF measurement for digital mammography quality control

Med. Phys. 32, 1684 (2005); 10.1118/1.1921667 


\title{
Development of an equipment for real time MTF measurement of optical systems
}

\author{
Dimas Rodrigues Romano*, Sergio Antonio de Almeida Nobre* and Bráulio \\ Fonseca Carneiro de Albuquerque ${ }^{\dagger}$
}

\author{
*OPTOVAC Mecânica e Optoeletrônica LTDA. - Osasco - SP - Brazil \\ ${ }^{\dagger}$ National Institute for Space Research, Space Engeneering and Technology, Aerospace Electronics Division - \\ SP - Brazil
}

\begin{abstract}
The quality of optical systems concerning contrast and resolution can be quantified through the use of the modulation transfer function (MTF) analysis. This metrology method can give us information about how much contrast is lost when light traverses an optical system for each spatial frequency until the cutoff, or Nyquist frequency. In this work is presented a procedure based on the measurement of a knife edge target from which one can extract the line spread function and, as a consequence, the optical transfer function needed to the MTF analysis. We used in the analysis a least square algorithm to fit the experimental data of the edge spread function and a FFT algorithm to extract the optical transfer function from the line spread function of the measured system. It is of great interest to apply this metrology analysis directly in lens production in order to have real measurements of quality for the optical components as they are manufactured. With this objective in mind we developed a MTF measurement equipment and we will talk about the difficulties involved, and its general characteristics. The main characteristic of our measurement equipment is the possibility of real time measurements, important in the fast quality control assurance needed in lens production.
\end{abstract}

Keywords: MTF, metrology

PACS: 06.20.-f, 42.87.-d

\section{INTRODUCTION}

The modulation transfer function (MTF) is widely used in characterization of optical systems concerning contrast and resolution. This function, normalized to unity for frequency zero, gives us information about how much contrast is transferred through an optical system for each spatial frequency until the cutoff frequency, and is generally used to certify the quality of such systems.

An input signal that is transmitted through an optical system is shown in Figure 1:
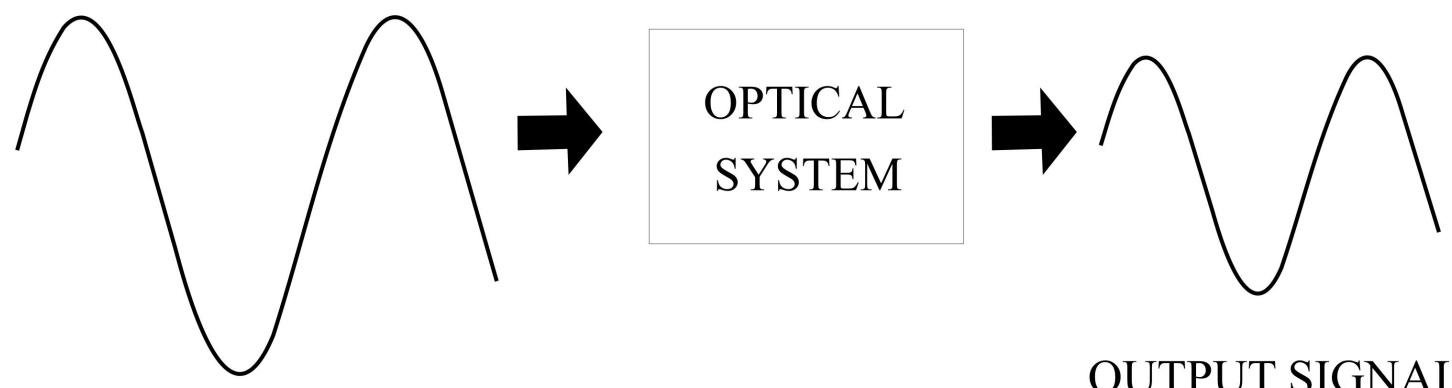

INPUT SIGNAL

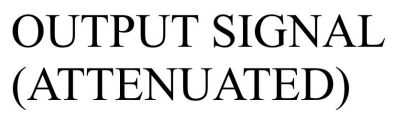

FIGURE 1. Signal through an optical system.

The modulation is a relation between the maximum intensity and the minimum intensity of the signal:

$$
M=\frac{I_{M A X}-I_{M I N}}{I_{M A X}+I_{M I N}}
$$


and the modulation fraction is given by:

$$
M T=\frac{M_{\text {OUTPUT }}}{M_{\text {INPUT }}}
$$

With this information taken for each spatial frequency we have the modulation transfer function of the optical system as shown in Figure 2.

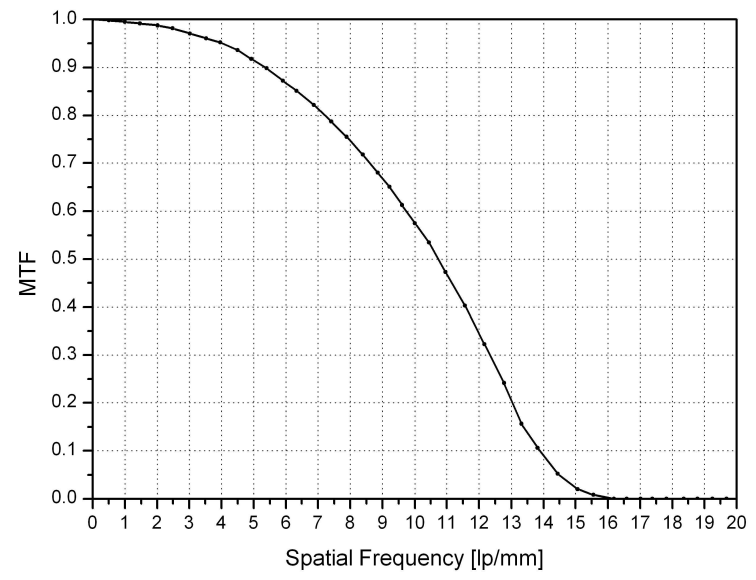

FIGURE 2. Example of a modulation transfer function.

The MTF is also related with the point spread function (PSF) of the system, which is an image of a point like source projected by the optical system at the image plane. This function is also known as the impulse response of the system. By applying a Fourier transform to the PSF the optical transfer function (OTF) of the system is obtained and the MTF is the modulus of the OTF.

There are several methods used to extract the MTF of an optical system each using different target geometries namely, sinusoidal patterns, point like sources, slits and knife edge targets $[1,2,3]$. Our equipment was developed to use a knife edge target and our software then process one dimensional functions extracted from the image (edge spread function) to determine the MTF of optical systems. All the one dimensional calculations can be done fast enough for the equipment to be considered a real time MTF measurement equipment.

It is important in lens production to have metrology methods that allow us to assure the quality of the optical components produced and MTF provides us with a quantitative and direct information about this quality. It can be used to analyze individual components and also help the assembly of precision optical systems, processes that require a fast analysis in order to be used in production.

In the following sections we will present the MTF measurement method used, the equipment developed and the difficulties involved in the process. This article is divided in the following sections. Section 2 discuss the equipment used. Section 3 discuss the method adopted. Section 4 finishes the work with the conclusions.

\section{EXPERIMENTAL SETUP}

The equipment is composed of an illumination source, a knife edge target, a collimator, a microscope objective, a CCD camera, and a computer to acquire and process the images. A diagram of the setup is shown in Figure 3. The back illuminated target is projected into the optical system under analysis and the resultant image is focused at the CCD array with the help of the microscope objective.

The MTF is sensible to some optical adjustments in the equipment namely, alignment between the knife edge target and the CCD array, focusing of the image plane in the CCD array, alignment of the components in the equipment, and intensity of the illumination.

If there exists an angle between the knife edge direction and the CCD array the edge spread function will have a width greater than the real one, as shown in Figure 4. 


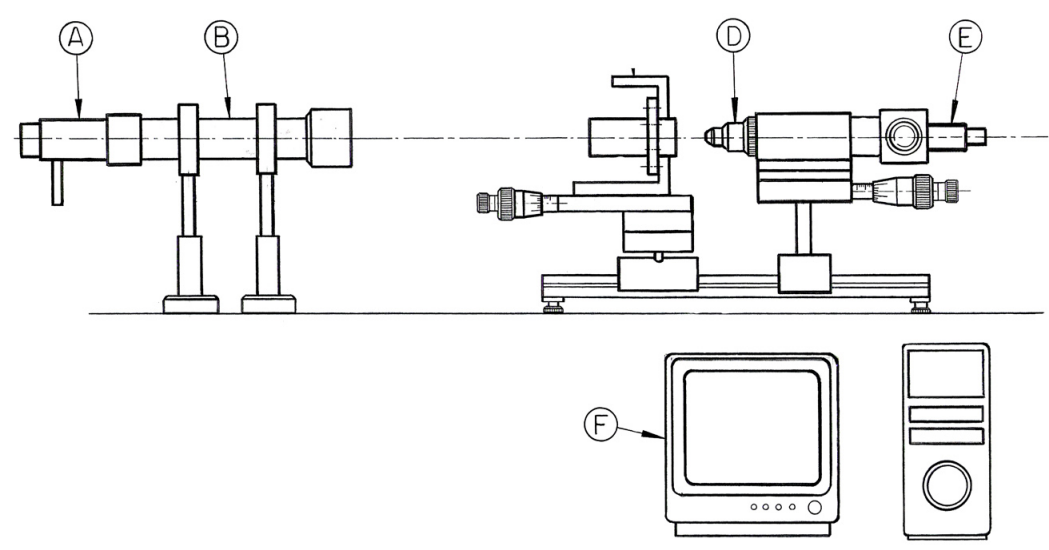

FIGURE 3. Diagram of the equipment. A) Illumination and knife edge target. B) Collimator. D) Microscope objective. E) CCD camera. F) Computer.

The consequence of this misalignment is a wider edge spread function that will result in an underestimated MTF. With our equipment we can perform this alignment manually, while acquiring measures, searching for the target position that results in the best MTF. It is important to notice that we chose this approach based on the fact that the real time characteristic of the equipment makes this kind of adjustment fast and easy. The correction of this misalignment could be done during the image processing and this angle between the target direction and the CCD array could also be used to oversample the image as discussed by reference [2,3], but we avoided extra calculations in the image processing and we do not have problems with undersampling as well.

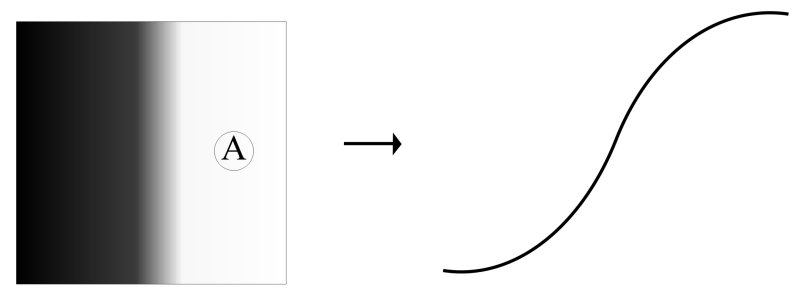

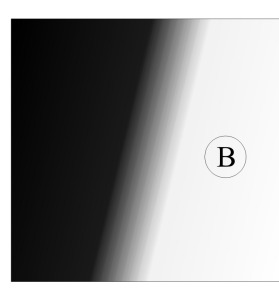

IMAGE

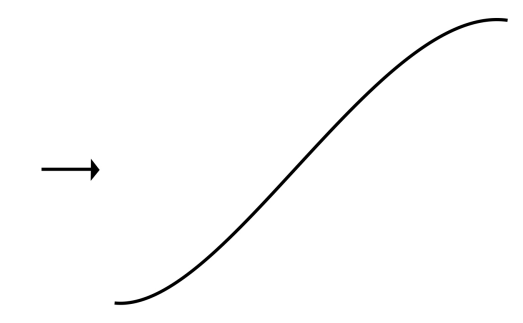

PROFILE

FIGURE 4. Effect of target misalignment. A) Aligned target and the respective edge spread function. B) Misaligned target and the respective edge spread function. In this situation the edge spread function is wider.

Focusing the image plane at the CCD array is also critical to the MTF analysis and this adjustment can also be done 
at real time in an easy way as described before. We perform the fine focus setting using a micrometric adjust at the base of the microscope objective/CCD camera device. Errors in this focus adjustment will cause a deterioration of the final MTF and can even invert the phase of the input signal.

Another adjustment that can be easily done using the real time characteristic of the equipment is the illumination intensity adjustment. The intensity requires a regulation to avoid saturation of the image acquired. We can adjust it checking the edge profiles shown real time by the software. Saturated images will cause fake MTF for the optical system.

And finally, the importance of the alignment of all components in the equipment is obvious. Tilts and centering errors also deteriorates the MTF.

This quick and easy way of dealing with critical adjustments makes the equipment suitable to be used in the production line. The MTF of samples can be easily measured during production.

\section{MTF ANALYSIS USING A KNIFE EDGE TARGET}

A variety of methods can be used to obtain a modulation transfer function for a determined optical system using different target geometries like sinusoidal patterns, point like sources, lines and knife edge targets. The first method compares the output amplitude with the input amplitude of a sinusoidal pattern to get the modulation transfer function while the others are based in finding the optical transfer function from the Fourier transform of a spread function of the system to be analyzed. In this work we use a one dimensional function, a profile extracted from the image of the knife edge target to realize the calculations and obtain the MTF.

\section{Knife edge target image acquisition}

Acquiring a knife edge target that will be used in MTF analysis is a task highly dependent on the sampling of the image. The CCD pixel size and the microscope objective magnification used together define the sampling rate of the image. We need to sample the image with at least twice the spatial frequency of interest for the measurement. this is a condition imposed by the Nyquist criterion [4], and following this criterion will guarantee no aliasing effects on the image sampling. With our equipment we sample the image with a spatial frequency of $600 \mathrm{lp} / \mathrm{mm}$ and as a consequence we are able to measure the MTF until a spatial frequency of $300 \mathrm{lp} / \mathrm{mm}$.

Another important fact in image acquisition is the alignment between the direction of the knife edge target and the CCD array, because the one dimensional profile of the knife edge is extracted from the image in the direction perpendicular to the direction of the edge, and as already explained this misalignment causes errors in the MTF.

\section{CCD calibration}

To minimize the errors present in image acquisition it is necessary to calibrate the CCD array. These errors are related with offset, random noise and quantum efficiency of the CCD pixels.

The offset correction is called DC current calibration. When no light strikes the CCD array we have a minimum value of the pixels that it is not zero. To correct this we need to acquire some dark frame images, images acquired with no light reaching the CCD, and generate an average dark frame (to reduce the random noise). We then subtract this average dark frame from all the frames acquired to correct the offset.

The correction concerning the difference in the quantum efficiency of different pixels is called flat field correction. First we need to acquire white images of homogeneous illumination approximately with half charge at the pixels. These various images are then used to generate an average white image (to reduce random noise). We then find a mean pixel value of this image and divide each pixel of the image by this mean pixel value. This procedure will result in a matrix of values, an "image" that will be used as a correction, the flat filed image. Each image acquired needs to be divided by this flat field image, pixel by pixel, and the result is an image flat field corrected.

The only procedure that minimizes the random noise is to work with average images, but this is not feasible if we wish a system capable of realizing real time analysis. A faster approach is to use a mean value from some lines of a given image.

It is important to notice that in order to diminish the number of calculations all this corrections can be done only in the lines of interest and not in the entire image. 
After acquiring the edge spread function we will use it to find the line spread function. This can be done by two methods.

1) Discrete derivative

We can obtain the line spread function of the system applying a discrete derivative on the experimental points of the edge spread function. However, the process of diferentiating the experimental points lowers the signal to noise ratio introducing errors that affect the final MTF. In using this method a correction must be applied to obtain the right MTF, as shown in [5]. This correction is unnecessary if the sampling is four times greater than the spatial frequency of interest.

2) Model based interpolation of the experimental data

The line spread function can also be obtained if we use a model function to interpolate the edge spread function experimental data. After the interpolation we can calculate an analytical derivative based on the interpolation parameters and use it as our line spread function. This is how we procedure in our work using a sigmoidal function [6] interpolated by the Leverberg-Marquardt least squares algorithm.

To obtain the optical transfer function of the system under analysis we now apply a Fourier transform on the line spread function. The fast fourier transform algorithm used in this work is the FFTW [7], widely used in this kind of analysis.

Finally we get the MTF from the modulus of the OTF and normalizes it to unity for spatial frequency zero.

\section{Optical system MTF}

In a linear optical system the total MTF equals the equipment MTF multiplied by the MTF of the system under analysis. To obtain the MTF of the system under analysis we need to do:

$$
M T F_{S Y S}=\frac{M T F_{T O T}}{M T F_{E Q U I P}}
$$

The measurement of the MTF of the equipment is made without the presence of the optical system. In this case the target is focalized directly in the CCD array with the help of the microscope objective, and the MTF of the equipment is acquired. This is a sort of calibration procedure and the equipment MTF will be used to correct the values of all subsequently measured MTF. It is necessary to acquire another MTF of the system each time a part of the equipment needs replacement. A change of the microscope objective or the CCD camera is an example.

Figure 5 shows an example of an optical system measured by our equipment. In this measurement we used an COSINA f28mm commercial objective to test the equipment and certify its functionality.

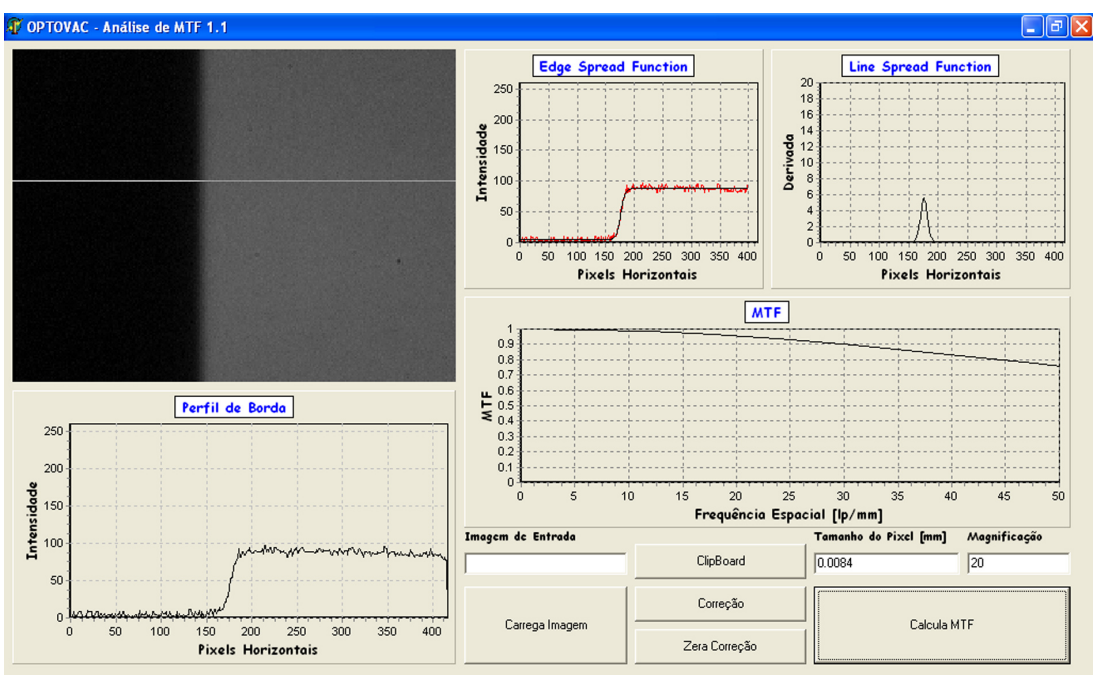

FIGURE 5. MTF measurement of a COSINA f28mm, with $\mathrm{f} \#=8$. 


\section{CONCLUSIONS}

The production of precision optics requires a metrology equipment that realizes the well known MTF analysis to assure the quality concerning contrast and resolution. In this work we have described the MTF analysis method adopted by us and the equipment developed to the experimental data acquisition. Even with all the CCD calibrations being applied to the images acquired, an interpolation of the experimental data by a sigmoidal function, and a fast fourier transform being performed on the line spread function generated by the interpolation, we could develop a complete equipment, experimental setup and processing software, that is able to realize real time MTF measurements of optical systems. This equipment is then suitable to be used directly in the production line of lenses and optical systems since all the critical adjustments can be done in a fast and easy way.

\section{REFERENCES}

1. J.M. Boone, T. Yu, and J.A. Seibert, Med. Phys. 23 (12), 1996, pp. 1955-1963.

2. P.B. Greer, and T. van Doorn, Med. Phys. 27 (9), 2000, pp. 2048-2059.

3. H. Fujita, D. Tsai, T. Ito, K. Doi, J. Morishita, K. Ueda, and A. Ohtsuka, IEEE Trans. on Medical Image Vol. II No. 1, 1992, pp. 34-39.

4. L. Lévesque, Eur. J. Phys., 22 (2001), pp. 127-132.

5. I.A. Cunningham, and A. Fenster, Med. Phys. 14 (4), 1987, pp. 533-537.

6. S. Blonski, M. Pagnutti, R.E. Ryan, and V. Zanoni, Proc. SPIE, Vol. 4814 (2002), pp. 317-326.

7. Fast Fourier Transform in the West, www. fftw.org. 